\title{
Electronic excitation processes in rare gas clusters studied by electron energy loss spectroscopy
}

\author{
Hayato KUBOTERA $^{1 *}$, Satoshi SAKAI ${ }^{1}$, Tatsuya SEKITSUKA ${ }^{1}$, Takayuki TACHIBANA ${ }^{2}$ \\ and Takato HIRAYAMA ${ }^{1,2}$ \\ ${ }^{1}$ Department of Physics, Rikkyo University, Toshimaku, Tokyo 171-8501, JAPAN \\ ${ }^{2}$ Research Center for Measurement in Advanced Studies (RCMAS), Rikkyo University, \\ Toshimaku, Tokyo 171-8501, JAPAN \\ *kubotera@rikkyo.ac.jp
}

\begin{abstract}
We present the electron energy loss spectra for Ar clusters as a function of incident electron energy and of cluster size. In spectra measured with $100 \mathrm{eV}$ incident electron energy the bulk excitation peak becomes visible for a mean cluster size above 170 atoms per cluster. For $250 \mathrm{eV}$ incident electron energy the bulk excitation peak is clearly observable even for a mean cluster size of 120 atoms per cluster. These experimental results are qualitatively reproduced by a simple calculation that accounts for the mean free path of electrons in Ar clusters; i.e., the penetration depth of incident electrons into the cluster.
\end{abstract}




\section{Introduction}

The physics of clusters has attracted significant interest because they not only bridge the gap between atoms and solids, but they have specific physical properties. One unique aspect of clusters is the large surface-to-volume ratio, which allows for sensitive measurements of surface processes. In addition, clusters traveling through a vacuum have very clean surfaces, which is not the case for a solid substrate surface; this is especially true for condensed solids such as rare gas solids. This property is important because it is known that small amounts of impurities may affect dynamic processes on the surface [1].

Many interesting properties of the electronic excitation processes for rare gas clusters have been revealed by numerous studies that employed synchrotron radiation. The electronic structure of rare gas clusters has been compared with that of atoms and solids and shown to vary with cluster size [2,3]. These results show that bulk electronic properties emerge above about 100 atoms per cluster. In addition, clusters have a specific electronic state, the so-called "cluster exciton," which is observed if the cluster radius is comparable to the mean radius of the electronically excited state [4]. The connection between the electronic and geometric structures has been studied using a core-level spectroscopic technique $[5,6]$.

Although many studies have used synchrotron radiation to investigate electronic excitation processes in rare gas clusters, few have performed electron-impact experiments [7, 8]. The advantages of such experiments compared with the photoabsorption technique are as follows:

(1) they allow observations of optically forbidden transitions, and

(2) the electron penetration depth is easily changed by varying the incident electron energy, making it possible to separately observe surface and bulk excitations.

The slightly lower resolution compared with the optical technique is not a large problem because clusters usually exhibit rather broad spectral features.

In the present paper, we report the electron energy loss spectra for Ar clusters as a function of incident electron energy and of cluster size. We show a stronger influence of the bulk properties with increasing cluster size. Dependence of the electron energy loss spectra on the incident electron energy is discussed in terms of the incident electron penetration depth.

\section{Experimental set-up}

Figure 1 shows a schematic diagram of the experimental setup, which consists of a cluster beam source, a differential pumping system, and an electron energy spectrometer. A pulsed cluster beam is generated by adiabatic expansion through a conical nozzle (inner diameter, 150 $\mu \mathrm{m}$; cone angle, $2 \theta=20^{\circ}$; length, $21 \mathrm{~mm}$ ) attached to a pulse valve (General Valve Co.). The relative position of the nozzle and skimmer, and their distance can be varied from outside the vacuum chamber to achieve optimum conditions. Typical duration and frequency of the cluster beam pulse are $10 \mathrm{~ms}$ and $2-4 \mathrm{~Hz}$, respectively. The nozzle temperature can be varied between $-100{ }^{\circ} \mathrm{C}$ and room temperature. The average cluster size is controlled by changing the stagnation pressure (2-5 atm) and nozzle temperature. The size distribution is determined by measuring the kinetic energy of the ionized cluster beam [9].

A monoenergetic electron beam is generated using a simulated hemispherical energy selector. [10] The electron beam is deflected by $90^{\circ}$ by a set of parallel plates, and then merges with the cluster beam. The length of the merging region is about $200 \mathrm{~mm}$. After passing through the merging region, the electrons are energy-analyzed and detected by a channel electron multiplier. The overall energy resolution of our setup is $150-180 \mathrm{meV}$.

To obtain the energy loss spectrum for Ar clusters, we measured two spectra at the same time: one measured during the period when the pulse valve is open, and the other with the valve closed. The open-valve spectrum contains the true (i.e., cluster) signals and the background signals. Only the background signals are accumulated in the closed-valve spectrum. The cluster spectrum is then obtained by subtracting the closed-valve spectrum from the open-valve spectrum. The intensity of the background signal is approximately 5-20\% of the true signal. We note that background signals due to unclustered monomers in the cluster beam cannot be excluded using this method. The amount of unclustered monomer in the cluster beam is experimentally estimated to be at most several percent, which is negligible in the case of the 
present measurements.

\section{Results and discussion}

Figures 2 and 3 show electron energy loss spectra of Ar clusters for average cluster sizes $\langle N\rangle$ ranging from 120 to 270 at incident electron energies $E_{0}=100 \mathrm{eV}$ (Fig. 2) and $250 \mathrm{eV}$ (Fig. 3 ). The spectrum of an Ar atom is also shown in Fig. 2. The observation angle is $0^{\circ}$ with respect to the direction of the incident electron beam. Vertical lines indicate the energy positions of the surface and bulk excitons in solid Ar $[11,12]$. The surface and bulk excitation peaks are clearly observed in the spectra. The intensity of the bulk excitation peak at $12.1 \mathrm{eV}$ increases with cluster size $\langle N\rangle$ in both spectra. The longitudinal branch [11] of the bulk exciton at $12.5 \mathrm{eV}$ is also observed for $\langle N\rangle \sim 270$ with $E_{0}=250 \mathrm{eV}$ (Fig. 3).

These results show that bulk excitation can occur in clusters with diameters corresponding to about 5 atoms, consistent with the results of Wörmer et al [13]. The bulk excitation peak becomes visible for cluster sizes $\langle N\rangle$ greater than 170 in the spectrum measured with $E_{0}=100$ $\mathrm{eV}$ (Fig. 2), and the bulk excitation peak is clearly observable as a shoulder at $12.1 \mathrm{eV}$, even for $\langle N\rangle \sim 120$ in the spectrum with $E_{0}=250 \mathrm{eV}$ (Fig. 3). This finding can be understood from the kinetic energy dependence of the mean free path $\lambda$ of the incident electrons in Ar clusters; i.e., the penetration depth of the incident electrons into the cluster. A similar idea has been successfully applied to photoelectron spectroscopy for rare gas clusters [14]. The mean free path $\lambda$ of incident electrons with energy $E_{0}=100 \mathrm{eV}$ and $E_{0}=250 \mathrm{eV}$ is about $0.7 \mathrm{~nm}$ and $1.2 \mathrm{~nm}$, [11] respectively. As the nearest neighbor distance of solid $\mathrm{Ar}$ is $0.376 \mathrm{~nm}$ [11], the bulk excitation is suppressed because of the smaller penetration depth for $E_{0}=100 \mathrm{eV}$ compared with that for $E_{0}=250 \mathrm{eV}$. The details of this effect are discussed in the next section.

The peak at $13.2 \mathrm{eV}$ is assigned to the $3 \mathrm{p}^{5} 4 \mathrm{p}$-type surface exciton in solid $\operatorname{Ar}[15,16]$, which is an optically forbidden state but is known to be partially allowed due to the lower spatial symmetry of the surface of clusters and solids. The intensity of this peak increases relative to that of the $3 p^{5} 4 \mathrm{~s}$-type surface exciton at $11.8 \mathrm{eV}$ with increasing cluster size, suggesting that the $13.2 \mathrm{eV}$ peak may originate from the bulk excitation. Hirayama and Arakawa [17] found that the $2 p^{5} 3 p$-type surface exciton in solid Ne shows a bulk feature similar to the present results for $\mathrm{Ar}$ clusters. They concluded that this type of exciton can be created at the surface and at lattice defects in the bulk. This reasoning can also be applied to the present case.

Wörmer et al. reported the energy shift of the bulk and surface excitons in Ar clusters as a function of cluster size [3]. The shift expected for the present results is about $10 \mathrm{meV}$, which is not observed because of the insufficient resolution of our spectrometer.

\section{Dependence of the surface and bulk excitation intensities on the incident electron energy}

We now discuss the dependence of the surface and bulk excitation intensities on the incident electron energy. The ratio of the number of surface atoms $N_{\mathrm{s}}$ to the total number of atoms $N$ in a cluster is given by [2]

$$
\begin{aligned}
& \frac{N_{S}}{N}=\left(\frac{4 \pi R^{2}}{\pi r^{2}}\right) /\left(\frac{4 \pi R^{3} / 3}{4 \pi r^{3} / 3}\right)=4 \frac{r}{R}=4 N^{-1 / 3}, \\
& \frac{N_{S}}{N} N^{1 / 3}=4,
\end{aligned}
$$

where $r$ and $R$ are the radius of an atom and a cluster, respectively. This equation shows that $\left(N_{\mathrm{s}} / N\right) N^{1 / 3}$ gives a constant value of 4 independent of cluster size.

Assuming that the intensity of surface excitation $I_{\mathrm{S}}$ is proportional to the number of surface atoms $N_{\mathrm{S}}$ and that the total intensity $I=I_{\mathrm{S}}+I_{\mathrm{B}}$ is proportional to the experimentally determined cluster size $\langle N\rangle$, we can write Eq. (2) as

$$
\frac{I_{S}}{I_{S}+I_{B}}<N>^{1 / 3}=4
$$


The peak intensities of the surface excitation $I_{\mathrm{S}}$ and those of the bulk excitation $I_{\mathrm{B}}$ are obtained by fitting the measured spectra to three Gaussian peaks, as shown in Fig. 4.

Figure 5 shows a plot of $\left(I_{s} / I\right)\langle N\rangle^{1 / 3}$ versus $\langle N\rangle^{1 / 3}$. The experimental results for $E_{0}=100$ $\mathrm{eV}$ (solid circles) and $250 \mathrm{eV}$ (open circles) show a value around 4 . The results also show that these values increase with increasing cluster size $\langle N\rangle$, and that the values for $E_{0}=250 \mathrm{eV}$ are smaller than those for $E_{0}=100 \mathrm{eV}$. We attribute this finding to the fact that the mean free path $\lambda$ of the incident electrons is smaller than the cluster diameter, and that $\lambda$ varies with the incident electron energy. In other words, the number of bulk atoms $N_{\mathrm{B}}$ ' that can be excited by an incident electron is smaller than the total number of bulk atoms $N_{\mathrm{B}}$.

We calculated $N_{\mathrm{B}}{ }^{\prime}$ using the following simple model. We use the fact that the nearest neighbor distance in solid Ar is $0.376 \mathrm{~nm}$, and that the mean free path of incident electrons with $E_{0}=100 \mathrm{eV}$ and $E_{0}=250 \mathrm{eV}$ in solid Ar is about $0.7 \mathrm{~nm}$ and $1.2 \mathrm{~nm}$, respectively. We assume that only atoms in the second layer from the cluster surface can be excited by incident electrons with $E_{0}=100 \mathrm{eV}$, and those in the second and third layers can be excited by incident electrons with $E_{0}=250 \mathrm{eV}$. We calculated for closed-shell icosahedra and the results are shown in Fig. 5. The number of atoms in each layer ( $n$th shell) $L(n)$ is calculated by [18]

$$
\begin{aligned}
L(n) & =1 & & (\text { for } n=1) \\
& =10 n^{2}-20 n+12 & & (\text { for } n \geq 2)
\end{aligned}
$$

Although the model calculation qualitatively reproduce the trend in the experimental results; i.e. $\langle N\rangle^{1 / 3}$ dependence and $E_{0}$ dependence, systematic differences between the calculation and experimental data are found. This discrepancy suggests that the electron-impact excitation for the cluster is more surface sensitive than expected from the simple geometrical consideration. Further discussion along this line, however, is not possible because of luck of the detailed information on the penetration depth (mean free path) of the electron in a cluster, the difference of the electron-impact excitation cross sections between the atoms on the surface and bulk, etc.

\section{Conclusion}

We studied the electron energy loss spectra for rare gas clusters as a function of incident electron energy and cluster size. These results clearly show the surface and bulk excitation peaks. The appearance cluster size of the bulk excitation peak is found to be dependent on the incident electron energy. This finding is explained in terms of the mean free path $\lambda$ of incident electrons in Ar clusters. The experimental results are compared with a simple calculation taking into account the mean free path of incident electrons in Ar clusters. The calculated results qualitatively reproduce the experimental results. The present study demonstrates the usefulness of electron energy loss spectroscopy in investigating electronic excitation processes in clusters.

\section{Acknowledgments}

The authors are grateful to Mr. Takanori Zen and Mr. Masaki Setone for their significant contributions in the initial stage of this work. We would also like to thank Prof. T. Koizumi for his continuous support throughout this work. This work was partly supported by the Rikkyo University Special Fund for Research.

\section{References}

[1] T. Kuninobu, A. Hayama, T. Hirayama, I. Arakawa, Surf. Sci. 390 (1997) 272.

[2] J. Stapelfeldt, J. Wörmer, T. Möller, Phys. Rev. Lett. 62 (1989) 98.

[3] J. Wörmer, R. Karnbach, M. Joppien, T. Möller, J. Chem. Phys. 104 (1996) 8269.

[4] M. Joppien, R. Müller, J. Wörmer, T. Möller, Phys. Rev. B 47 (1993) 12984.

[5] F. Federmann, O. Björneholm, A. Beutler, T. Möller, Phys. Rev. Lett. 73 (1994) 1549.

[6] O. Björneholm, F. Federmann, F. Fössing, T. Möller, Phys. Rev. Lett. 74 (1995) 3017.

[7] A. Burose, C. Becker, A. Ding, Z. Phys. D 20 (1991) 35.

[8] M. Allan, J. Phys. B: At. Mol. Opt. Phys. 26 (1993) L73.

[9] T. Hirayama, A. Kanehira, I. Arakawa, Rev. Sci. Instrum. 64 (1993) 962.

[10] K. Jost, J. Phys. E 12 (1979) 1006. 
[11] N. Schwentner, E. E. Koch, J. Jortner, Electronic Excitations in Condensed Rare Gases, Springer-Verlag, Berlin, 1985.

[12] M. Michaud, L. Sanche, Phys. Rev. B 50 (1994) 4725.

[13] J. Wörmer, M. Joppien, G. Zimmerer, T. Möller, Phys. Rev. Lett. 67 (1991) 2053.

[14] M. Tchaplyguine, R. R. Marinho, M. Gisselbrecht, J. Schulz, N. Mårtensson,

S. L. Sorensen, A. Naves de Brito, R. Feifel, G. Öhrwall, M. Lundwall, S. Svensson,

O. Björneholm, J. Chem. Phys. 120 (2004) 345.

[15] V. Saile, M. Skibowski, W. Steinmann, P. Gürtler, E. E. Koch, A. Kozevnikov, Phys. Rev. Lett. 37 (1976) 305.

[16] T. Kloiber, G. Zimmerer, Phys. Scr. 41 (1990) 962.

[17] T. Hirayama, I. Arakawa, J. Phys. Cond. Matt. 18 (2006) S1563.

[18] M. R. Hoare, Adv. Chem. Phys. 40 (1979) 49. 


\section{Figure Captions.}

Fig. 1. Schematic view of the experimental set-up.

Fig. 2. Electron energy loss spectra for Ar clusters with $\langle N\rangle=120,170$ and 270 . The incident electron energy is $100 \mathrm{eV}$ and the observation angle is $0^{\circ}$. Vertical lines show the energy position of surface and bulk excitons in solid Ar [10]. The spectrum of an Ar atom is also shown.

Fig. 3. Electron energy loss spectra for Ar clusters with $\langle N\rangle=120,170$ and 270 . The incident electron energy is $250 \mathrm{eV}$ and the observation angle is $0^{\circ}$. Vertical lines show the energy position of surface and bulk excitons in solid Ar [10].

Fig. 4. Electron energy loss spectrum of Ar clusters from 11 to $13 \mathrm{eV}$. $I_{\mathrm{S}}$ and $I_{\mathrm{B}}$ are the intensities of surface and bulk excitations, respectively. See text for details.

Fig. 5. Plot of $\left(I_{\mathrm{S}} / I\right)\langle N\rangle^{1 / 3}$ vs. $\langle N\rangle^{1 / 3}$. Solid and open circles are experimental results for $E_{0}=$ $100 \mathrm{eV}$ and $E_{0}=250 \mathrm{eV}$, respectively. Dotted and solid lines are calculated results for closed-shell icosahedra taking into account the mean free path of incident electrons. See text for details. 


\section{Differencial}

pumping

Main chamber
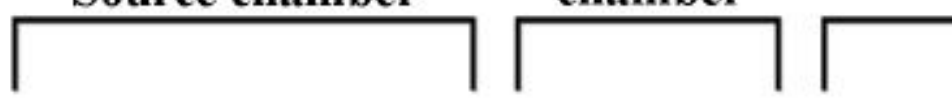

Energy selector

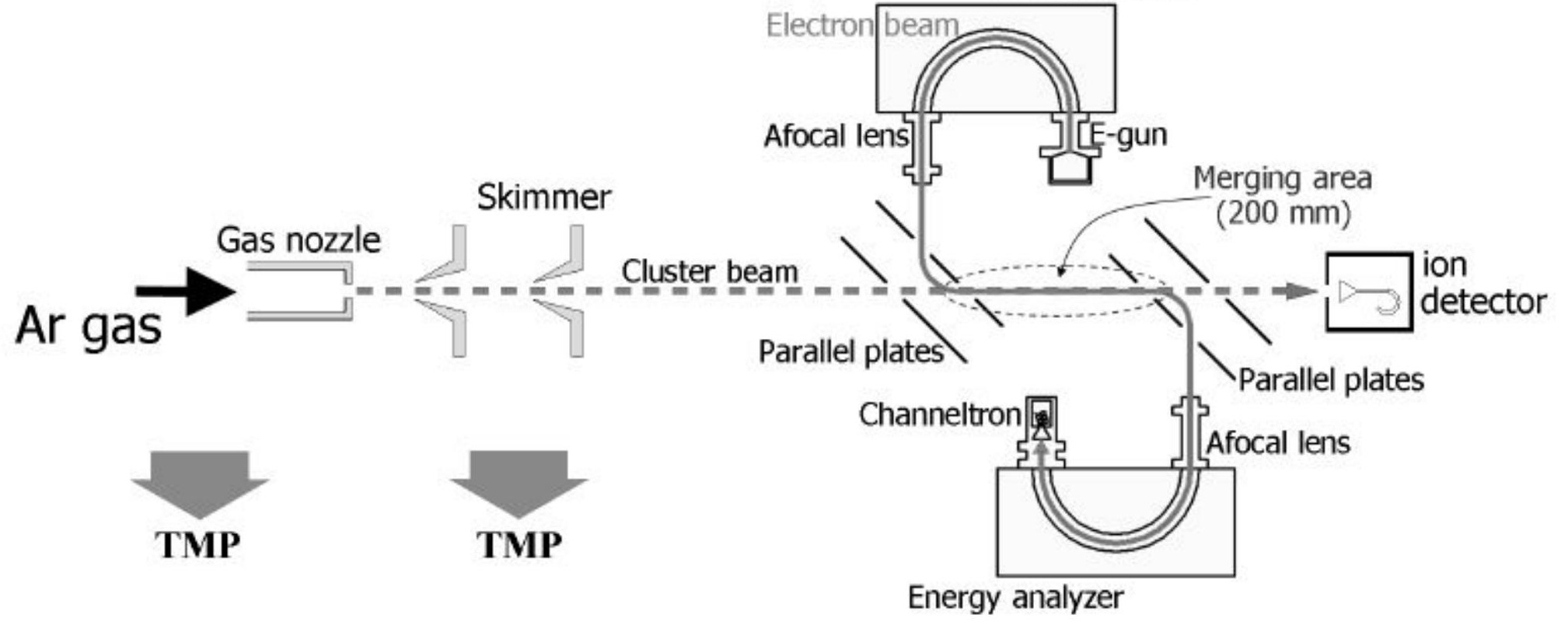




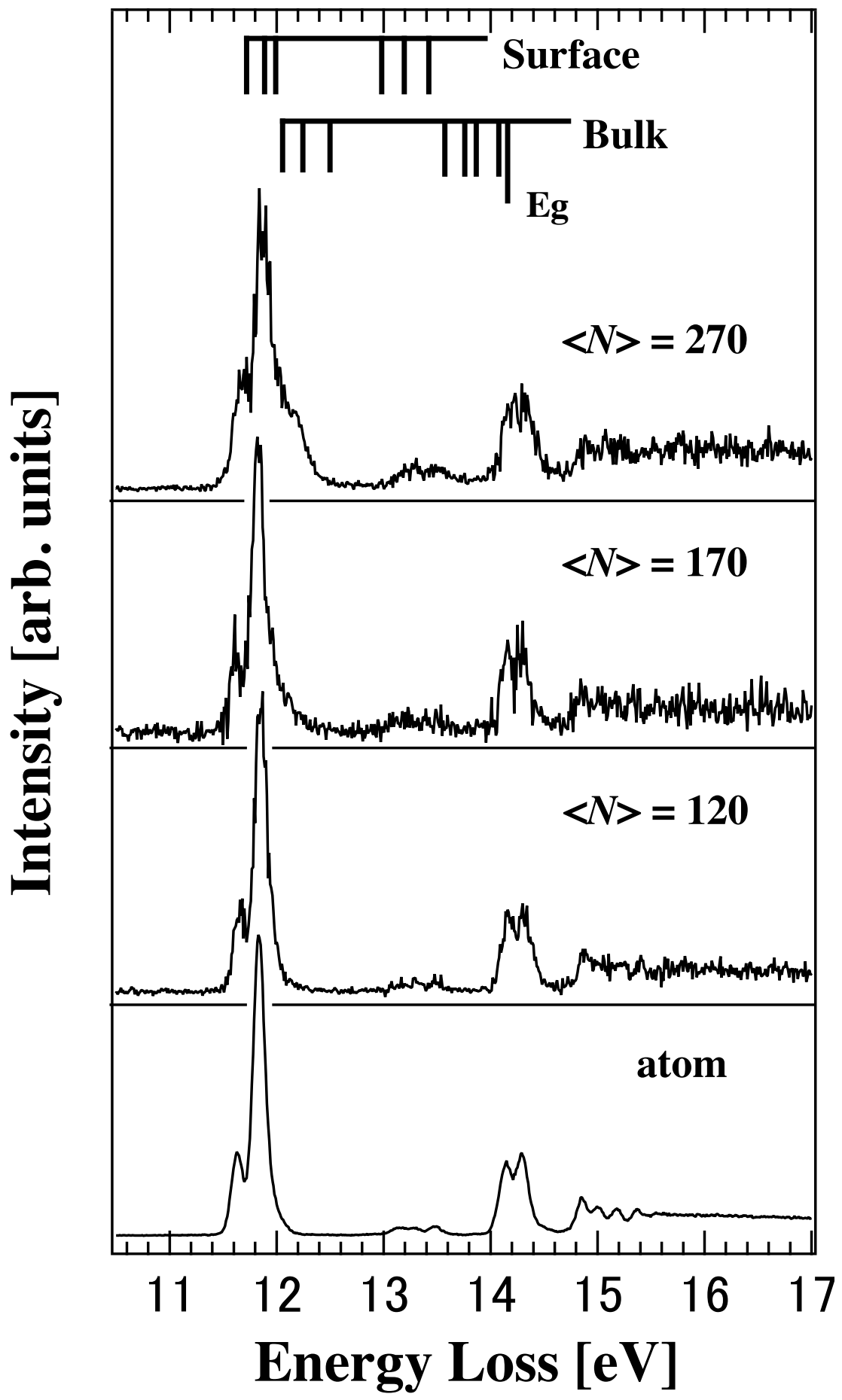




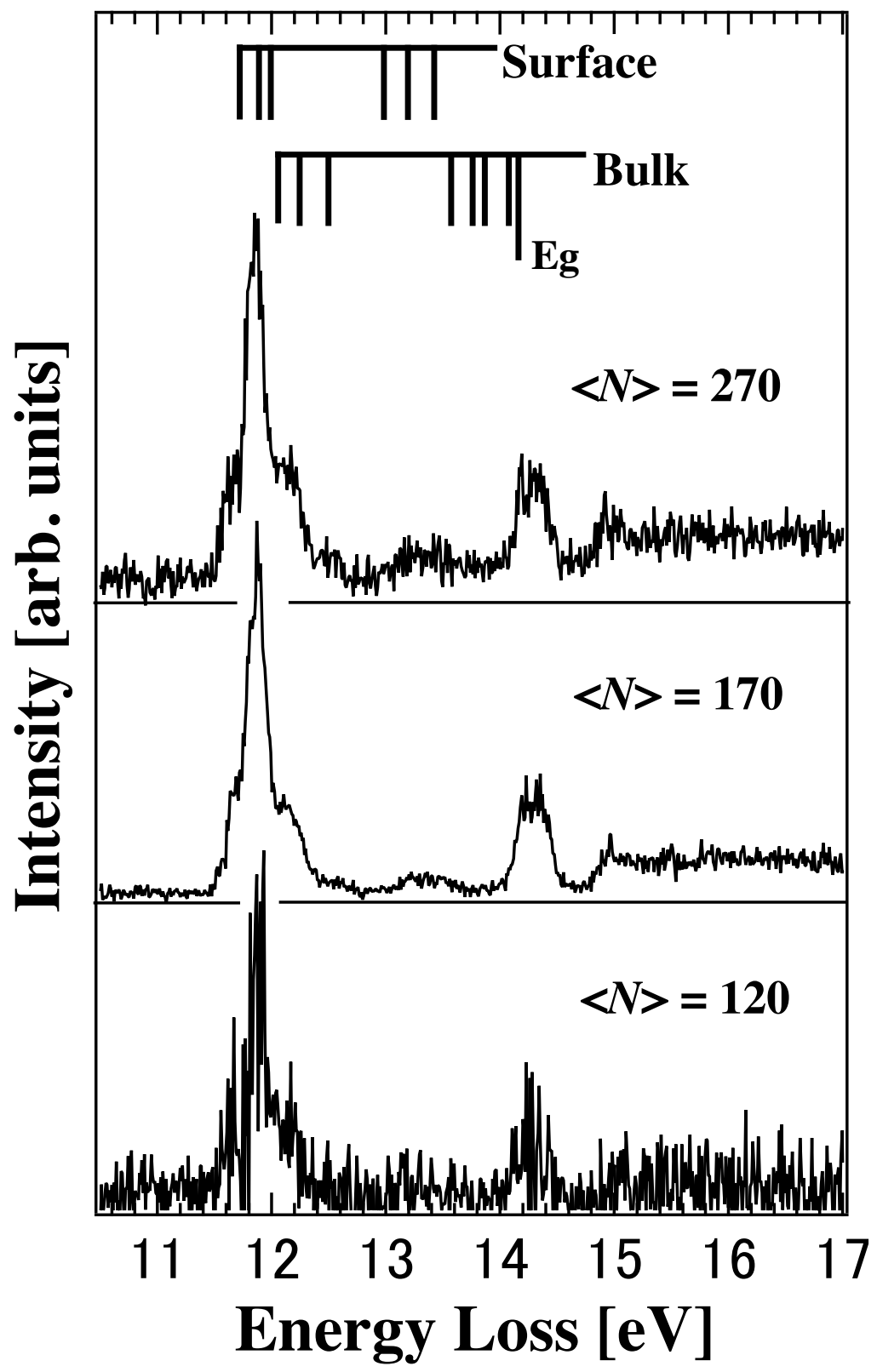




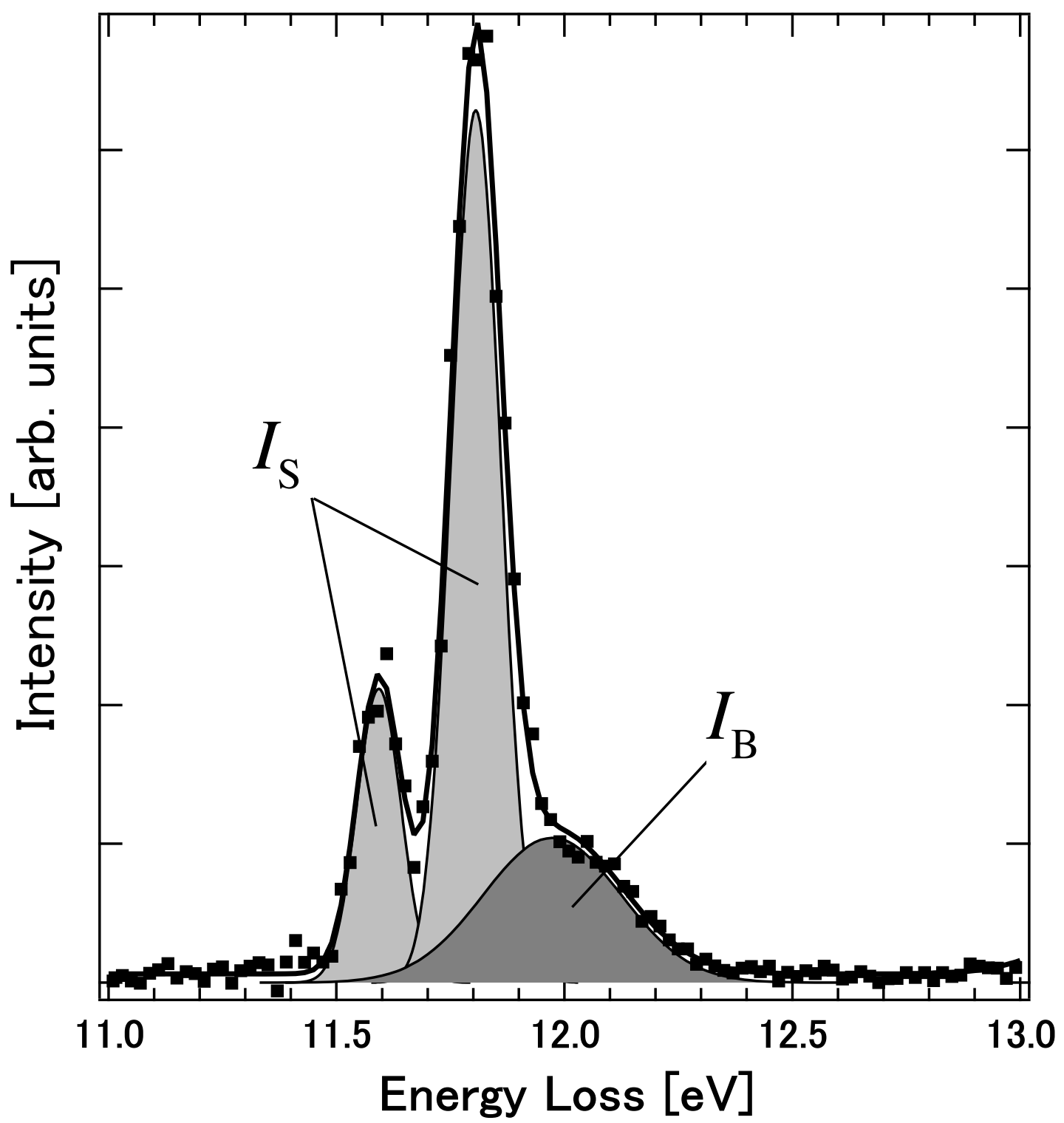




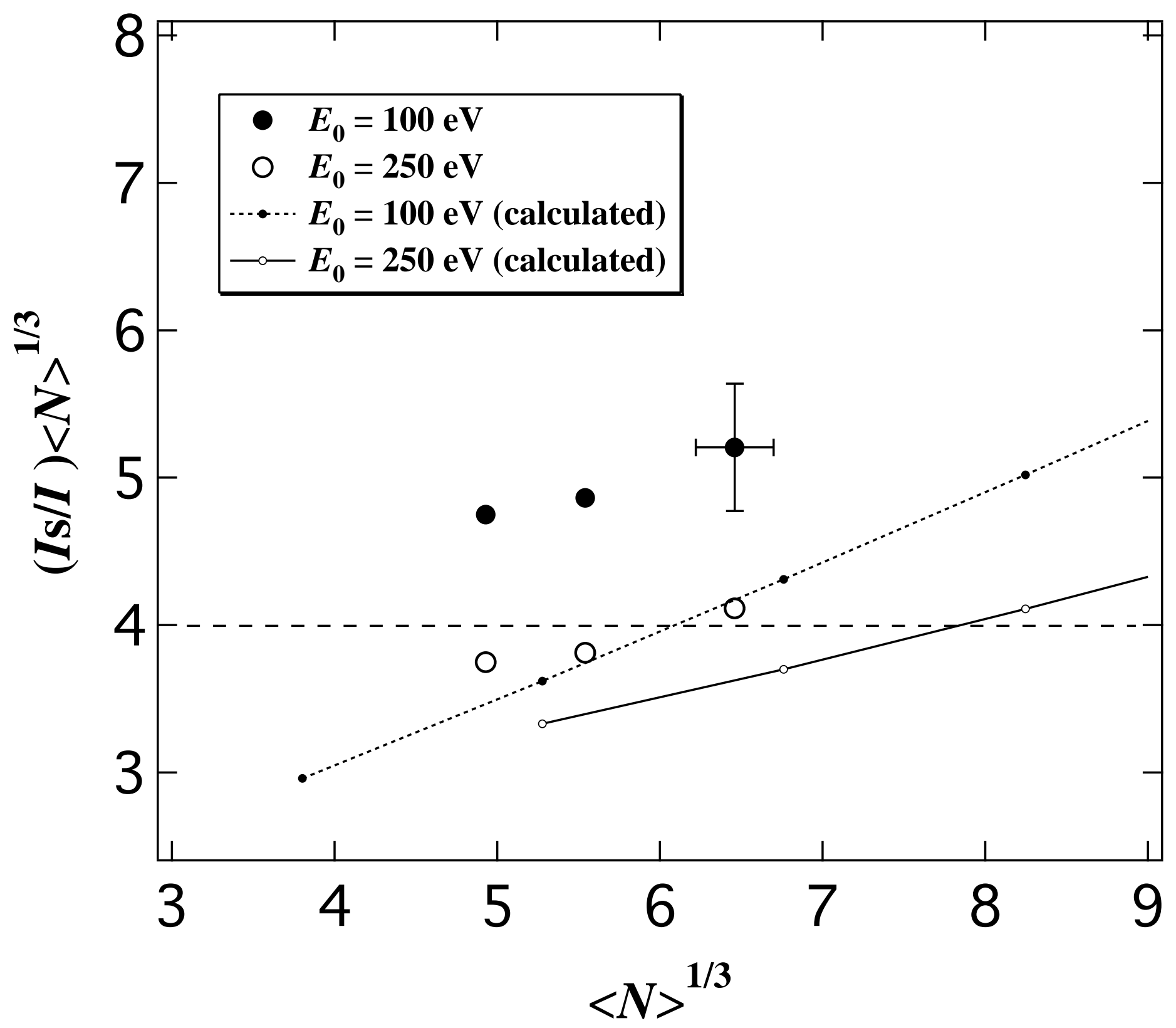

